\title{
Charles River Sprague Dawley Rats Lack Early Age-Dependent Susceptibility to DMBA-Induced Mammary Carcinogenesis
}

\author{
R.B. Gear ${ }^{1}$, M. Yan ${ }^{1}$, J. Schneider ${ }^{1}$, P. Succop ${ }^{3}$, S.C. Heffelfinger ${ }^{2}$, and D.J. Clegg ${ }^{1}$ \\ 1. Department of Psychiatry, University of Cincinnati, PO Box 670559, Cincinnati, OH 45267-0559, USA \\ 2. Department of Pathology, University of Cincinnati, Cincinnati, Ohio, USA \\ 3. Department of Environmental Health, University of Cincinnati, Cincinnati, OH 45267-0056, USA
}

Correspondence to: Deborah J. Clegg, Ph.D., Assistant Professor, University of Cincinnati, Department of Psychiatry, Genome Research Institute, Obesity Research Center, 2170 East Galbraith Road, Cincinnati, OH 45237. U.C. M.L. 0506. Voice: 513-558-3429; Fax: 513-558-4800; E-mail: debbie.clegg@uc.edu

Received: 2007.04.16; Accepted: 2007.10.03; Published: 2007.10.04

Developmental stages of mammary glands influence their susceptibility to initiating events related to carcinogenesis. The "window of susceptibility" to mammary carcinogenesis is classically defined as the time in early puberty when the mammary gland morphology is most sensitive to initiation events. Administration of the polyaromatic hydrocarbon, 7,12-dimethylbenz(a)anthracene (DMBA), in a single oral dose yields maximal mammary tumor formation when administered in this "window". We examined the DMBA treated mammary glands, precursor lesions, and morphology of the uninvolved mammary epithelium for the first 100 days of life for Charles River Sprague Dawley CDR IGS. Our goal was to determine the DMBA dose at which $50 \%$ of the rats (IC50) developed carcinoma in situ (CIS) within three months of dosing. Here we demonstrate, rather than the classical U-shaped dose curve in which there is maximum sensitivity for DMBA at 50 days, there is an increasing degree of sensitivity with age in the CD ${ }^{\mathrm{R}}$ IGS rat. Additionally, we report that vehicle-treated animals developed mammary CIS without any known initiator, and 100 day virgin animals demonstrated lactational changes, independent of DMBA exposure or dose. Lastly, we demonstrate this strain of virgin female rats has elevated pituitary prolactin immunoreactivity independent of the level of mammary differentiation. We conclude this strain of Charles River Sprague Dawley rats has prolactin-induced pituitary stimulation, and therefore, the window of susceptibility for mammary tumorigenesis is absent.

Key words: DMBA, mammary carcinogenesis, Charles River Sprague Dawley rats

\section{Introduction}

Research on rodent mammary gland carcinogenesis and observational studies in human populations has focused on the premise that the breast has a unique age at which carcinogen exposure is maximally affective. This 'window of susceptibility' is thought to be a major predictor of cancer risk.

Extensive comparisons have been made between rat and human mammary pathologies $[1 ; 2]$. The rat mammary chemical carcinogenesis model has been extremely well studied and is commonly used to ask questions about mechanisms of tumorigenesis throughout the lifespan of the animal. One of the major questions regarding mammary carcinogenesis is the mechanism by which mammary glands at different stages of development display a differential sensitivity to initiating events. Mammary gland development begins with a single lactiferous duct giving rise primitive ductules at birth [3]. The lobule begins as a terminal end bud (TEB), which differentiates into alveolar buds $(\mathrm{AB})$. $\mathrm{AB}$ develop to become the terminal ductolobular unit (TDLU), containing the stem cells from which all TDLU cells are derived [4]. Detailed analysis of human tumors shows that the site of origin is the TDLU or the lobule [5]. The "window of susceptibility" to mammary carcinogenesis is classically defined as the time in early puberty in which there is maximum burst of TEB proliferation and development into $\mathrm{AB}$ [6; 7].

Administration of the polyaromatic hydrocarbon, 7,12-dimethylbenz(a)anthracene (DMBA), in a single oral dose yields maximal mammary tumor formation when administered in this "window" [8]. Among strains, the outbred Sprague Dawley (SD) rats are among the most sensitive to carcinogen-induced mammary cancers [4;9]. The early work identifying the mammary window of susceptibility showed that the peri-pubescent period of about 45-50 days of age was consistently found to be the time of optimal sensitivity for initiation with the model polyaromatic hydrocarbon DMBA [6; 8]. This model is well-known for the production of tumors that are morphologically heterogeneous and hormone dependent.

A number of studies have highlighted the fact that there is genetic strain divergence in rodent breeding colonies [10-13]. Therefore, we undertook the task of identifying the time span within the first 100 days of life in which the Charles River Sprague Dawley $\mathrm{CD}^{\mathrm{R}}$ IGS rat that is most susceptible to mammary 
carcinogenesis. The Charles River Sprague Dawley $\mathrm{CD}^{\mathrm{R}}$ IGS is known to have come to Charles River in 1950 from Sprague Dawley Inc. In 1991, 8 colonies were selected to form the IGS Foundation Colony using the Charles River International Genetic Standard System. Our research goal was to calculate a dose of DMBA at each age in which $50 \%$ of the rats developed carcinoma in situ (CIS) within three months (IC50) in this strain of Sprague Dawley female rats.

\section{Methods}

\section{DMBA Treatment and Tissue Isolation:}

Virgin female Sprague Dawley CD (CDR IGS) rats were purchased at three different ages: weaning (21 days), peri-pubertal (45 days), or mature (100 days) from Charles River Laboratories (all shipments were from either the Portage, MI or Raleigh, NC, breeding facilities). Upon arrival from Charles River the SD CD rats were placed on AIN-93G or AIN-93M defined diet (Bio-Serv, Inc.), absent phyoestrogens, and wood chip bedding. Animals were kept on a strict 12 hour light cycle to maintain circadian rhythm. For carcinogenesis studies, DMBA (Fisher Acros, Fisher Scientific) was dissolved in filter sterilized corn oil at $(2-15 \mathrm{mg} / \mathrm{mL}$ depending on BW dissolved at 95-96 C) cooled to room temp and administered with an enterral feeding tubing (Vygon, Inc.) under light isoflurane anesthesia. Based upon historical published studies [14-21], we chose the following DMBA dosages as a single oral gavage for each age under examination: 21 days old: 20, 50, and $100 \mathrm{mg} / \mathrm{kg}$; 50 days old: 10,30, and $60 \mathrm{mg} / \mathrm{kg}$; and 100 days old: 25,50 , and $80 \mathrm{mg} / \mathrm{kg}(\mathrm{n}=20$ for each dose and each age). Corn oil was administered by gavage for the vehicle control group $(n=5)$. Animals were palpated weekly for tumor formation. Three months post-DMBA exposure, all animals were sacrificed. At this time, all tumors and uninvolved mammary tissue were saved by formalin fixation for paraffin embedding and later histological characterization. Masses and precursor histology were identified as previously published by our laboratory $[14 ; 22 ; 23]$ and according to the criteria of the Russos [24].

By the Russo criteria, an intraductal proliferation initiated (IDPi) is the very earliest form of ductal hyperplasia in the DMBA model, whereas intraductal proliferation promoted (IDPp) is a form of ductal hyperplasia with cellular atypia [4]. These proliferative lesions occur in clusters and patches within lobules, so each lobule containing one or more alveolus with IDPp is counted as one. CIS is the next in situ stage of disease. CIS is often found within a benign adenoma or fibroadenoma, in which case the tumor is called CIS. Pure adenomas or fibroadenomas are counted as benign tumors. Once a malignant tumor has broken through the epithelial basement membrane and is invading adjacent stroma it is termed invasive (INV). INV is always derived from CIS, as previously noted [14]. Photomicrographs of each diagnostic category from our laboratory have been previously published [14]. Data from the current studies were compared to historical data from our laboratory in Sprague Dawley rats purchased from Taconic in which DMBA was administered at 50 days of age.

\section{Mammary Morphological Characterization}

In order to characterize the morphology of the mammary gland at the time of DMBA exposure, additional rats were acquired from Charles River (Portage, MI) at 21, 50, and 100 days of age, three per group. At sacrifice, mammary gland four was prepared for whole mount analysis. Abdominal-inguinal mammary tissue containing glands 4,5 , and 6 were excised in one piece and pinned to boards for fixation in $10 \%$ alcoholic formalin. After complete defatting with acetone, tissues were hydrated using a series of alcohols for 1 hour each and left in water overnight prior to staining for 2 hours with $0.025 \%$ toluidine blue. Once staining was complete, the whole mount preparations were destained using methanol and 70\% ethanol and fixed briefly in ammonium molybdate and rehydrated. Finally xylene was used to clear the tissue preparations. Tissues were mounted on slides using permount for further examination and analysis.

All whole mount images were captured using a Q Color 5 digital camera (Olympus, Tokyo Japan) into digital images. Using the QCapture Pro software, measurements were made detailing the size and complexity of gland 4. Area, area of epithelium, distance from nipple to lymph node, distance from superior lymph node to branch border, and distance from nipple to first lateral branch were all measured. Individual structures in defined regions of the ductal extensions into the mammary fat pad of abdominal gland 4 commonly known as zone $\mathrm{C}$ were counted according to the criteria of Russo and Russo [8] at 50 and 100 days. Structures from throughout the gland were counted at 21 days. Classic descriptive criteria [3] as well as the following measurement criteria were used for counting structures: TEB $>85$ microns and TD 58-84 microns. Approximately one thousand structures per animal were counted at 108X (Olympus, Tokyo Japan). Of the remaining glands, half were formalin fixed for histology. Pituitaries were fixed in formalin and paraffin embedded for histological and immunohistochemcial analysis.

\section{Immunohistochemistry}

All immunohistochemistry was performed using Ventana XT automatic immunostaining system, , with the Ventana I view DAB detection kit as described previously [14]. Preimmune Rabbit IgG or isotype-specific mouse IgG was added in place of the primary antibody as negative controls. Histologic sections were stained by immunohistochemistry for prolactin (monoclonal antibody Immunotech, clone 16422-12, 1:400 dilution with Ventana protease I digest) and PCNA (Vecter, clone pc10, 1:800 dilution with Ventana cell conditioner I mild). Rat adsorbed anti-mouse (Vector Laboratories, Burlingame, CA) was used as the secondary antibody at a 1:50 dilution.

\section{Statistical methods}

All statistical evaluations were performed using SigmaStat (SPSS, Chicago, Illinois). Group mean 
comparisons were by one-way ANOVA, two sided, using $95 \%$ confidence intervals. Two group comparisons were by Student's $t$ test.

\section{Results}

\section{Charles River Sprague Dawley Female Rats Lack the 'Traditional' Window of Susceptibility to DMBA-Induced Carcinogenesis}

The percentage of rats with CIS plus INV (tumors) for each DMBA dose at each age of exposure is shown in Figure 1. Benign tumors (adenoma and fibroadenomas) are excluded. Note that the DMBA dose is not directly comparable among cohorts by age. In the 21 and 50 day cohorts incidence shows a dose dependent response, reaching $100 \%$ incidence at the highest dose given. The 100 day cohort lacks a dose response curve and the maximum incidence reached was $80 \%$. Of note, the control animals from the 100 day cohort had an incidence of CIS of $20 \%$. At the time of sacrifice these rats were between six and six and a half months old; a young age for spontaneous mammary tumor formation.

Incidence of CIS and INV at All Ages

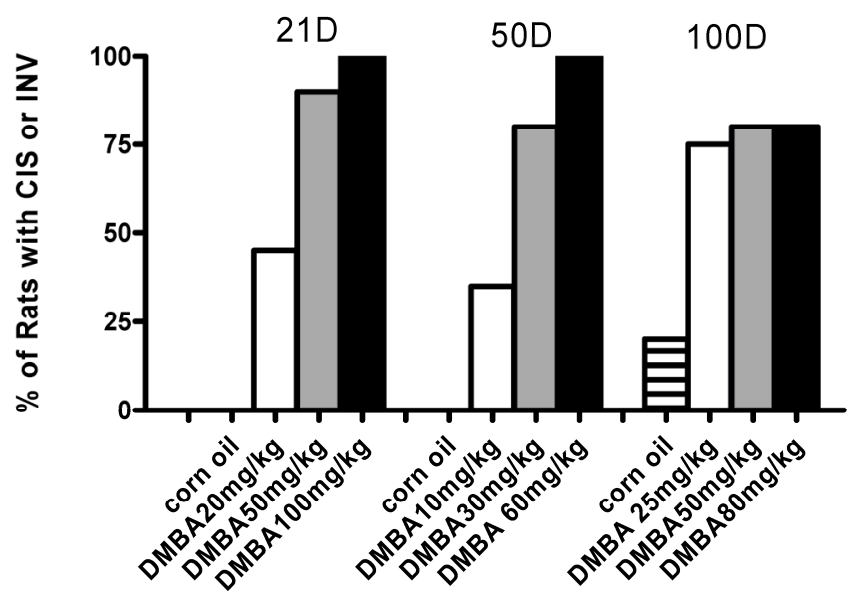

Figure 1. Percent of rats with CIS or INV when treated with different doses of DMBA at various ages either 21, 50 or 100 days. Plotted is the percent of rats with either CIS or INV for each group, determined 3 months post-DMBA exposure. For all ages, $n=5$ rats (corn oil) and $n=20$ for each DMBA treated group.

Based upon these data we could calculate the DMBA concentration necessary to induce tumors in $50 \%$ of the rats for each age group (IC50) from probit curves fit to the data. These results are shown in Table 1 as IC50 with $95 \%$ confidence intervals.

Table 1. Calculated IC50 for Charles River SD

\begin{tabular}{|c|c|c|}
\hline Age at Initiation (days) & IC50 $(\mathrm{mg} / \mathrm{kg})$ & $95 \% \mathrm{CI}$ \\
\hline 21 & 21.86 & $18.80-25.41$ \\
\hline 50 & 14.03 & $11.65-16.85$ \\
\hline 100 & 5.11 & $2.22-10.59$ \\
\hline
\end{tabular}

Historical reports suggest that DMBA exposure at times other than the 50 day old optimum leads to an increased frequency of benign tumors. Table 2 shows the data for each DMBA dose and time of exposure for

all tumor types. The benign category includes either fibroadenoma or adenoma with no malignant component. The CIS category includes CIS with no benign component. Mixed is CIS within a benign tumor. INV is invasion derived from CIS; we have not yet encountered an invasive DMBA-induced tumor in any study that was not clearly related to an adjacent CIS. As with the dose response for all other DMBA-induced pathologies, benign lesions increased with DMBA dose at all ages. However, there was no relationship between frequency of benign or mixed pathologies and age of DMBA exposure.

Table 2. Tumor Distribution by Type in Percent (and Total Count)

\begin{tabular}{|c|cccc}
\hline DMBA (mg/kg) & Benign & Mixed & CIS & INV \\
\hline A & & \multicolumn{2}{|c}{21 Days } \\
\hline $0($ oil $)$ & $0(0 / 0)$ & $0(0 / 0)$ & $0(0 / 0)$ & $0(0 / 0)$ \\
20 & $0(0 / 35)$ & $9(3 / 35)$ & $91(32 / 35)$ & $0(0 / 35)$ \\
50 & $2(2 / 105)$ & $17(18 / 105)$ & $81(85 / 105)$ & $0(0 / 105)$ \\
\hline 100 & $1(1 / 157)$ & $7(11 / 157)$ & $87(137 / 157)$ & $5(8 / 157)$ \\
\hline
\end{tabular}

\begin{tabular}{c|ccc|c|} 
B & \multicolumn{4}{|c}{50 Days } \\
\hline 0 (oil) & $0(0 / 0)$ & $0(0 / 0)$ & $0(0 / 0)$ & $0(0 / 0)$ \\
\hline 10 & $0(0 / 11)$ & $9(1 / 11)$ & $82(9 / 11)$ & $9(1 / 11)$ \\
\hline 30 & $2(2 / 65)$ & $3(3 / 65)$ & $94(61 / 65)$ & $2(2 / 65)$ \\
\hline 60 & $3(5 / 168)$ & $18(30 / 168)$ & $78(131 / 168)$ & $1(2 / 168)$
\end{tabular}

C

\begin{tabular}{|c|c|c|c|c|}
\hline 0 (oil) & $0(0 / 0)$ & $0(0 / 0)$ & $100(1 / 1)$ & $0(0 / 0)$ \\
\hline 25 & $0(0 / 91)$ & $1(1 / 91)$ & $97(88 / 91)$ & $2(2 / 91)$ \\
\hline 50 & $0(0 / 81)$ & $2(2 / 81)$ & $95(77 / 81)$ & $2(2 / 81)$ \\
\hline 80 & $1(1 / 183)$ & $10(18 / 183)$ & $84(154 / 183)$ & $6(10 / 183)$ \\
\hline
\end{tabular}

Charles River Sprague Dawley Female Rat Mammary Gland Morphology

To further characterize the nature of the carcinogenic process, we examined the mammary glands from each rat for precursor lesions and the morphology of the uninvolved mammary epithelium. The percentage of rats per group with IDPi, IDPp, CIS, and INV is shown in Figures 2a-c. Note all stages of pathology were identified at all doses at all ages. The 21 and 50 day cohorts show a dose response for each precursor lesion. INV is rare in all cohorts being most common at higher dosages. This is expected given that analyses are performed only three months post-DMBA exposure. Note that occasional precursor pathologies are found in the corn oil exposed 50 day and 100 day cohorts. The mean multiplicity per group for each pathology is shown in Figures 2d-f. There is a rough dose dependence for multiplicity of proliferative lesions and CIS among all groups. The corn oil control group in the 100 day cohort also had a reasonable frequency of proliferative lesions. 


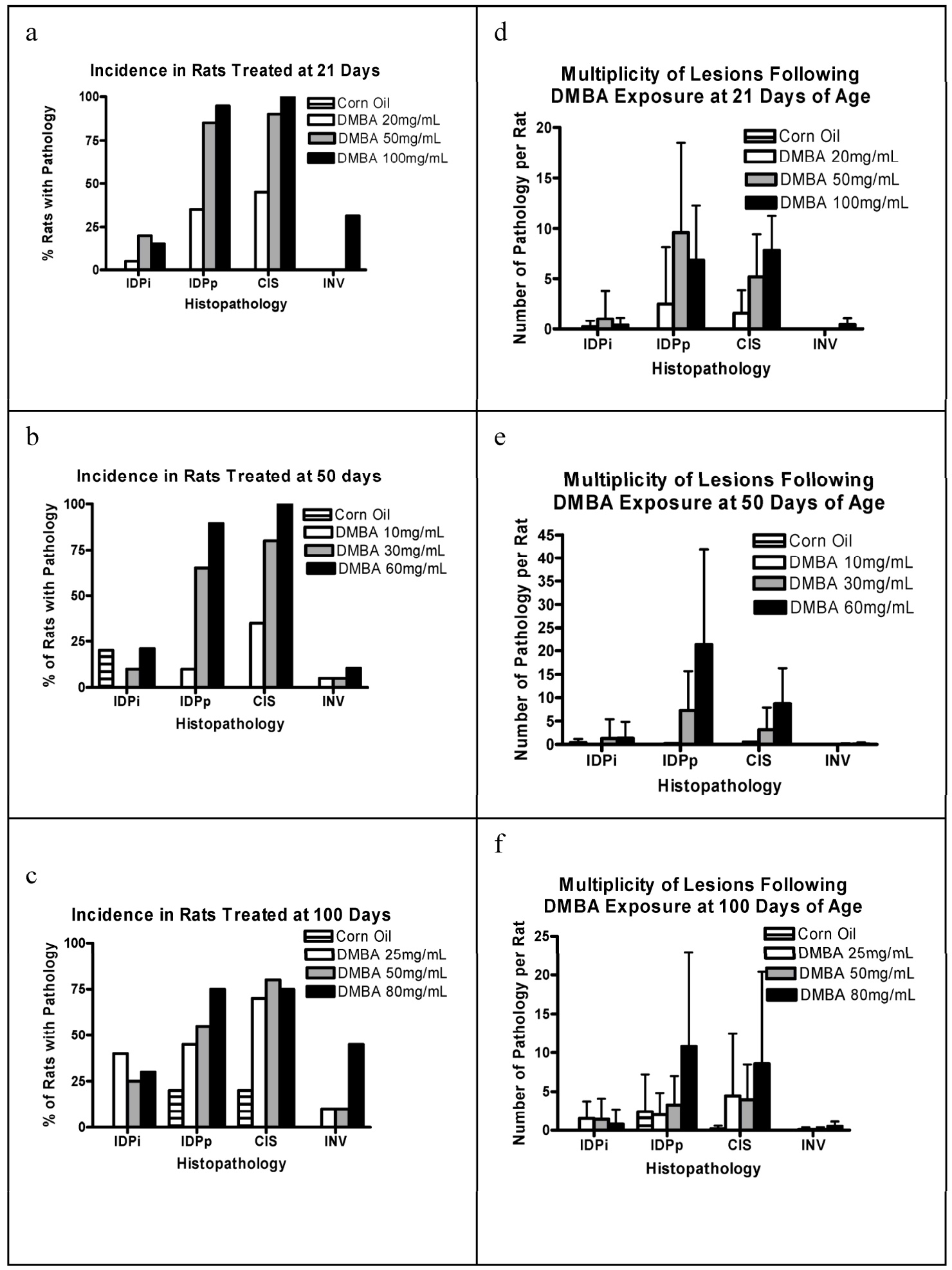

Figure 2. (a) Percent of rats with incidence of various pathologies when treated with 0 (corn oil), 20, $50 \mathrm{and} 100 \mathrm{mg} / \mathrm{kg}$ BW DMBA at 21 days of age; (b) at 50 days of age; (c) at 100 days Plotted is the percent of rats with each pathology for each treatment group, determined 3 months post-DMBA exposure for $n=5$ rats (corn oil) and $n=20$ for each DMBA treated group. (d) is the number of pathologies per rat when treated with 0 (corn oil), 20,50 and $100 \mathrm{mg} / \mathrm{kg} \mathrm{BW} \mathrm{DMBA}$ at 21 days of age; (e) at 50 days, and (f) at 100 days. Plotted is the mean + s.d. number of each pathology per rat for each group, determined 3 months post-DMBA exposure for $n=5$ rats (corn oil) and $n=20$ for each DMBA treated group. 


\section{Charles River Sprague Dawley Female Rats Have Increase Susceptibility to Pituitary Hyperplasia, Adenomas, and Spontaneous Carcinomas}

Figure 3 shows the types of histology found within the mammary glands of the 100 day cohort exposed only to a single oral gavage of corn oil three months prior to sacrifice. The mammary glands varied from lobules with milk-filled cystic spaces (as indicated by arrows) with fat globules (Figure 3a), lobules that were massively expanded with milk (indicated by * as in Figure 3b), and milk filled ducts (Figure 3c). Numerous animals with highly milk-expanded ducts were found in all treatment groups at this age.

In order to better understand the mammary gland morphology of the 100 day cohort with respect to the milk-filled cystic spaces, we examined the pituitary glands from female Sprague Dawley CDR IGS rats obtained from Charles River Laboratories. Immunohistochemical staining for prolactin in the 100 day animals showed a higher percentage of lactotrophs. An example of the histology from each strain is shown in Figure 4 (a and b). Panel (a) represents a low magnification view of the pituitary gland from a 100 day old representative vehicle control female rat; all other rats at that age showed a similar level of positive prolactin staining. Although not quantitative, these data suggest that lactotroph hyperplasia is present early in adult life and may be responsible for the lactational events seen at six months of age. By contrast, matched tissue from a representative vehicle control female Taconic CD (b) of the same age showed minimal prolactin secretion.

\section{Charles River CD IGS Rats Have Accelerated Mammary Gland Development}

Given the morphological correlates related to the early mammary gland susceptibility, we examined rat mammary gland 4 whole mounts for developmental characteristics at the ages in which we had given DMBA (21, 50 and 100 days). These data are summarized in Figure 5 . As predicted by the lactational phenotype, at 100 days of age there is significantly advanced lobular maturation in the mammary gland with numerous type 2 lobules and even a few type 3 lobules, reminiscent of a pseudopregnant state. All of the animals at 100 days of age had fluid filled lobules and fat globules within the epithelium, but none had undergone the proliferation necessary to form fully lactational type 4 lobules.

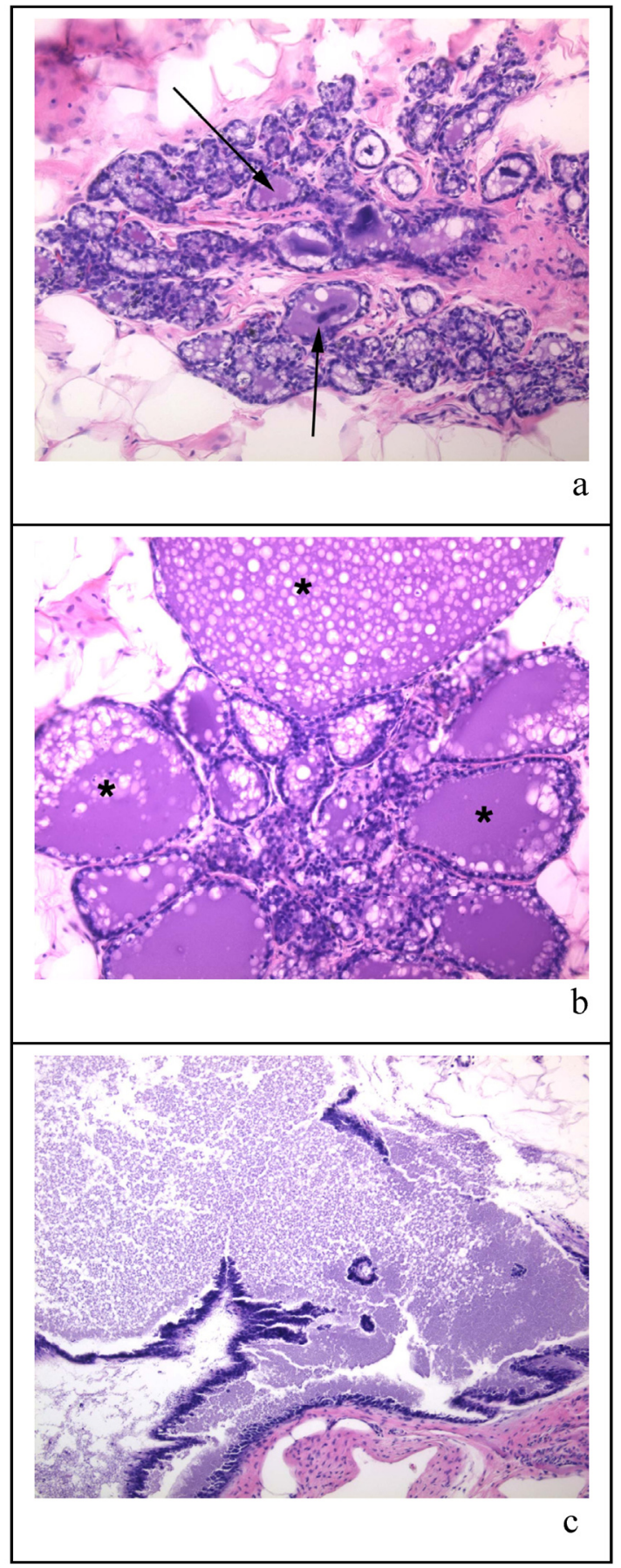

Figure 3. Panels (a) (b) and (c) are representative samples of histological sections of mammary glands from virgin corn oil treated rat at time of sacrifice (approx. 190 days old). The tissue sections are stained with $\mathrm{HE}$ and all are $\mathrm{x} 20$ magnification. The arrows in panel (a) indicate milk-filled cystic spaces; in panel (b) the * indicates specific lobules which are massively expanded by milk; panel (c) shows milk filled ducts. 


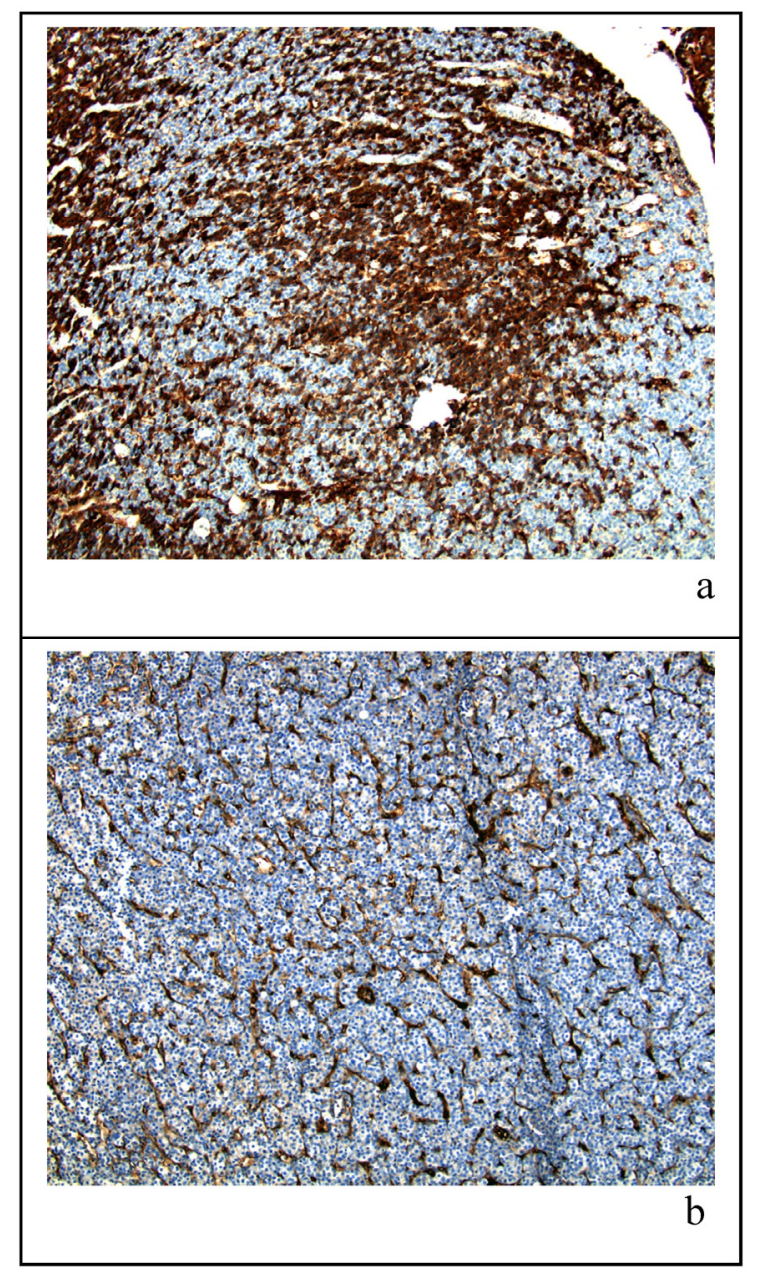

Figure 4. Panels (a) and (b) show histological sections of pituitary glands confirmed by hematoxalin and eosin staining (not shown) from 100 day old virgin rats dosed with vehicle then sacrificed at approximately 190 days of age. The tissue sections are stained for prolactin and both are at a $10 \mathrm{X}$ magnification. Panel (a) is taken from a Charles River Sprague-Dawley rat while panel (b) is from a Taconic Sprague-Dawley rat.

\section{Discussion}

Many studies examining genetic and promotional events in mammary carcinogenesis utilize the Sprague Dawley rat. Based upon our previous experience with Sprague Dawley rats from Taconic and previously published data in this strain, three DMBA doses were chosen for administration at weaning (21 days), 50 days, and 100 days of age. The expectation was that the most sensitive period for DMBA initiation would be the 50 day cohort with higher concentrations of DMBA required to reach a $50 \%$ incidence for tumors at younger and older ages in which one would predict fewer sensitive structures. Surprisingly, rather than the classical U-shaped dose curve in which there was maximum sensitivity for DMBA at 50 days, there appeared to be an increasing degree of sensitivity with age, quite the opposite of any other data published, to date. Based on these data, the corn oil cohort became of some interest due to the clear presence of a carcinogenic process, absent any known initiator. Furthermore, at sacrifice of the cohort exposed to DMBA at 100 days of age, the majority of the animals were lactating, independent of DMBA exposure or dose.

Because of these unexpected findings, we consulted with Charles River Laboratories regarding the Sprague Dawley CDR IGS breeding colonies and discovered that this outbred strain has a significant incidence of pituitary adenomas and mammary carcinomas. Data on their website (www.criver.com) compiled from 31 studies, reports that in two year old females, there is a $71 \%$ incidence of pituitary adenomas, $6 \%$ incidence of pituitary carcinomas, and a $22 \%$ incidence of spontaneous mammary carcinomas. If the pituitary pathology in this strain occurs early and the adenomas are prolactin producing, it would explain the lactational morphology and the early incidence of mammary tumors we observed in control rats. At some time in the past in the Charles River breeding stock, an increased susceptibility to pituitary hyperplasia, adenoma, and carcinoma developed. Prolactin is a major promoter of mammary tumorigenesis independent of the level of mammary differentiation, [25] and may even overcome the inhibitory effect of preganancy-induced terminal differentiation of the mammary gland [26]. Our data show that on this background of pituitary stimulation, the window of susceptibility for mammary tumorigenesis disappears in this rat strain.

The classical window of susceptibility for mammary carcinogenesis correlates with the highest density of TEB. In the SD rat this is usually about 45-50 days of age. In older rats, the TD is also reported to be a carcinogen sensitive structure. Carcinogens given before or after this period will yield fewer tumors. The reasons for this heightened sensitivity are still under study by numerous groups and may involve physiological factors and mammary gland stem cell populations that are uniquely present at this age. At the classical age of maximum sensitivity to carcinogenesis, 50 days, the Charles River animals had already undergone differentiation into type 1 and 2 lobules with about $10 \%$ each residual TEB, TD, and $\mathrm{AB}$. This is in contrast to the predicted morphology at this age in which TEB are rapidly being converted into $\mathrm{AB}$ [4]. Carcinogen sensitivity at this age correlates most directly with TD formation. Interestingly, at this time of weaning, the Charles River animals already had a significant number of type 1 lobules. The Charles River animals by 100 days of age show lactational changes, consistent with a pseudopregnant state. To our knowledge no one has published carcinogen sensitivity in this strain of outbred Sprague Dawleys in recent years. Here we have the opportunity to examine the correlation of carcinogen sensitivity and mammary structure on the background of prolactin stimulation. Our data suggest that pathologies that induce a change in physiological status, such as pituitary hyperplasia, alter the timing of maximum sensitivity to carcinogenic insults. Indeed, in older animals pituitary 
hormones are sufficiently promotional that a few percent of animals develop mammary tumors. In the Charles River line of SD rat, these pituitary events most likely explain the dose curve analyses showing a rapidly diminishing dose of carcinogen necessary to induce mammary carcinogenesis beginning at weaning.

Recently, there has been a considerably renewed interest in mammary gland morphology and timing of development as data have shown that in utero and perinatal hormonal exposures may change the rate and timing of maturation [27-29]. In order to assess alterations in lifetime risk to agents in the environment, one must be aware of how optimal timing of sensitivity may differ by agent and the sensitive period may vary due to environmental factors. For example, if an exposure moves the timing of $T E B$ to $A B$ formation to a younger age than the classical 45-50 day window, an analysis of sensitivity at that age using DMBA may falsely show a preventive effect, whereas, in fact, one has simply moved the most sensitive period to a younger age. Therefore, characterization of mammary carcinogenesis risk must be analyzed on the background of how the agent under study or other confounding influences alters the rate of mammary gland maturation.

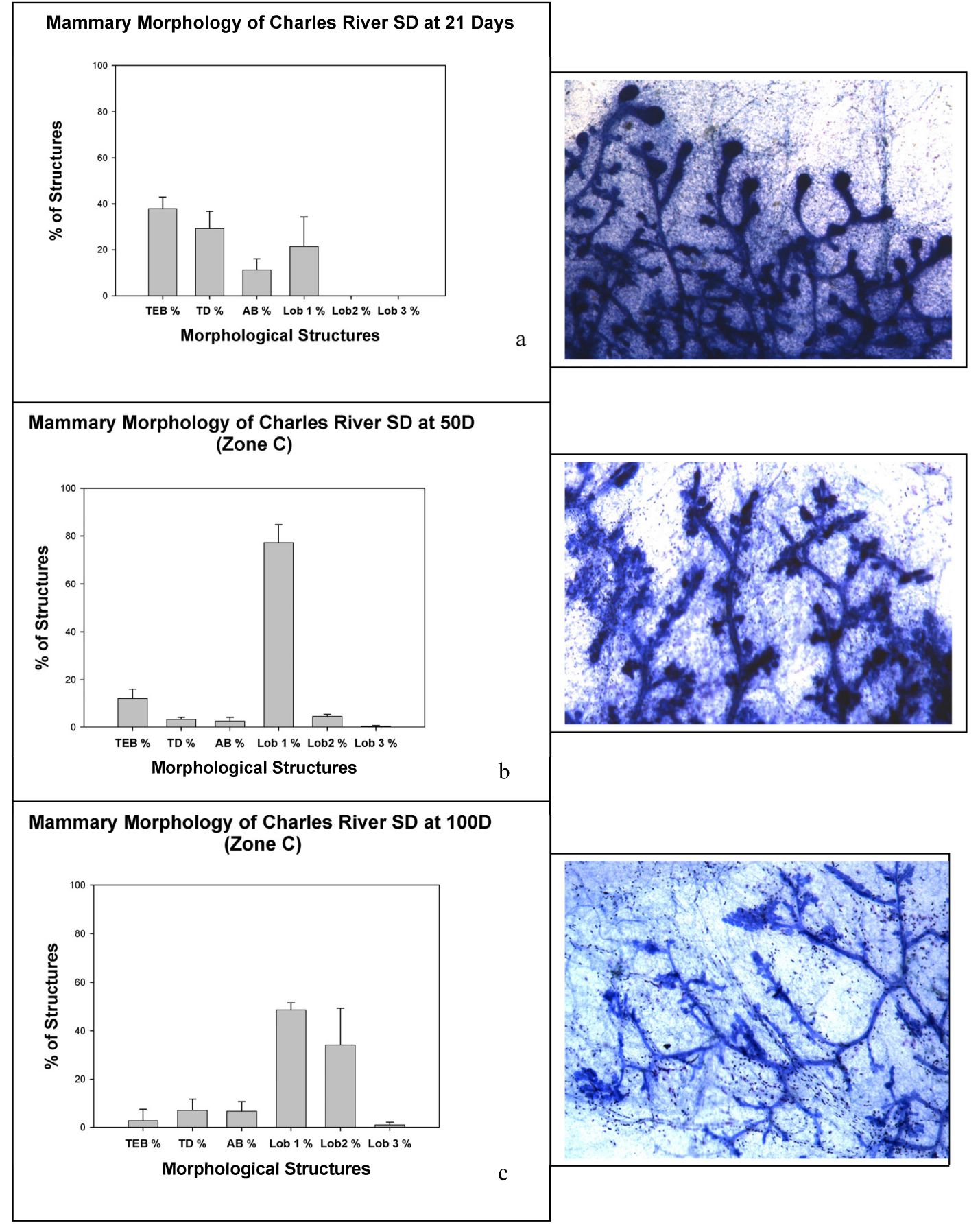

Figure 5. Panels (a), (b), and (c) show mammary morphology from animals at (a) 21 days, (b) 50 days, and (c) 100 days. 


\section{Summary}

The DMBA dose curves reported here highlight the difficulty in assuring physiological characteristics of animal strains over time. At some time in the past in the Charles River breeding stock, an increased susceptibility to pituitary hyperplasia, adenoma, and carcinoma developed. This has significantly altered the physiology of these animals such that pituitary hormones elicit early lactational events in virgins and promote mammary tumors in animals at a young age absent known carcinogenic insults. We briefly examined the pituitaries for prolactin, but clearly other pituitary hormones may also be over-produced in this strain.

\section{Acknowledgements}

The authors would like to thank Julie Bland for her excellent technical support in immunohistochemistry. This publication was made possible by the Breast Cancer and the Environment Research Centers grant number U01 ES/CA 012770-04 from the National Institute of Environmental Health Sciences (NIEHS), and the National Cancer Institute (NCI), NIH, DHHS. Its contents are solely the responsibility of the authors and do not necessarily represent the official views of the NIEHS or NCI, NIH.

\section{Conflict of interest}

The authors have declared that no conflict of interest exists.

\section{References}

1. Russo J, Gusterson B, Rogers A, Russo I, Wellings S, van Zwieten M. Biology of Disease: Comparative study of human and rat mammary tumorigenesis. Lab Invest 1990; 62:244-278

2. Singh M, McGinley J, Thompson H. A comparison of the histopathology of premalignant and malignant mammary gland lesions induced in sexually immature rats with those occurring in the human. Lab Invest 2000; 80:221-231

3. Russo J, Tay LK, Russo I. Differentiation of the mammary gland and susceptibility to carcinogenesis. Breast Cancer Res and Treat $1982 ; 2: 5-73$

4. Russo I, Russo J. Mammary gland neoplasia in long-term rodent studies. Environmental Health Perspectives 1996; 104:938-966

5. Wellings S, Jensen H, Marcum R. An atlas of subgross pathology of the human breast with special reference to possible precancerous lesions. J Natl Cancer Inst. 1975; 55:231-273

6. Huggins C, Grand L, Brillantes F. Mammary cancer induced by a single feeding of polynuclear hydrocarbons, and its suppression. Nature 1961; 189:204-207

7. Russo J, Russo I. Influence of differentiation and cell kinetics on the susceptibility of the rat mammary gland to carcinogenesis. Cancer Res, 1980, 40:2677

8. Russo IH, Russo J. Developmental stage of the rat mammary gland as determinant of its susceptibility to 7,12-dimethylbenz(a)anthracene. J Natl Cancer Inst 1978; 61:1439-1442

9. Isaacs J. Genetic control of resistance to chemically induced mammary adenocarcinogenesis in the rat. Cancer Res 1986; 46:3958-3963

10. Wieland S, Boren J, Consroe P, Martin A. Stock differences in the susceptibility of rats to learned helplessness training. Life Sci 1986; 39:937-944

11. Ciccotosto G, Hand T, Mains R, Eipper B. Breeding stock-specific variation in peptidylglycine alpha-amidating monooxygenase messenger ribonucleic acid splicing in rat pituitary. Endocrinology 2000; 141:476-486

12. Klinger M, MacCarter G, Boozer C. Body weight and composition in the Sprague Dawley rat: comparison of three outbred sources. Lab Anim Sci 1996; 46:67-70

13. Pettersen J, Morrissey R, Saunders D, Pavkov K, Luempert Lr, Turnier J, Matheson D, Schwartz D. A 2-year comparison study of Crl:CD BR and Hsd:Sprague-Dawley SD rats. Fundam Appl Toxicol 1996; 33:196-211

14. Heffelfinger S, Gear R, Schneider J, LaDow K, Yan M, Lu F, Pyle A, Warshawsky D. TNP-470 Inhibits DMBA-Induced Mammary Tumor Formation When Administered Prior to the Formation of CIS but is not Additive with Tamoxifen. Lab Invest 2003; 83:1001-1011

15. Heffelfinger S. Carcinogenesis of Breast Tumors. In: Landolph J, Warshawsky D, eds. Molecular Carcinogenesis. CRC Press. 2004.

16. Thompson HJ, Singh M. Rat models of premalignant breast disease. J Mammary Gland Biol Neoplasia 2000; 5:409-420

17. el-Bayoumy K, Chae YH, Upadhyaya P, Meschter C, Cohen LA, Reddy BS. Inhibition of 7,12-dimethylbenz(a)anthracene-induced tumors and DNA adduct formation in the mammary glands of female Sprague-Dawley rats by the synthetic organoselenium compound, 1,4-phenylenebis(methylene)selenocyanate. Cancer Res 1992; 52:2402-2407

18. Appelt LC, Reicks MM. Soy induces phase II enzymes but does not inhibit dimethylbenz[a]anthracene-induced carcinogenesis in female rats. J Nutr 1999; 129:1820-1826

19. Aylsworth CF, Cullum ME, Zile MH, Welsch CW. Influence of dietary retinyl acetate on normal rat mammary gland development and on the enhancement of 7,12-dimethylbenz[a]anthracene-induced rat mammary tumorigenesis by high levels of dietary fat. J Natl Cancer Inst 1986; 76:339-345

20. Sinha D, Pazik J, Dao T. Progression of rat mammary development with age and its relationship to carcinogenesis by a chemical carcinogen. Int J Cancer 1983; 31:321-327

21. Russo J, Wilgus G, Russo I. Susceptibility of the mammary gland to carcinogenesis. Am J Pathol 1979; 96:721-736

22. Heffelfinger SC, Gear RB, Taylor K, Schneider J, LaDow K, Miller MA, Warshawsky D. DMBA-induced pre-invasive mammary tumors are angiogenic in vitro and in vivo. Lab Invest 2000; 80:485-492

23. Heffelfinger S, Yan M, Gear R, Schneider J, LaDow K, Warshawsky D. Inhibition of VEGFR2 Prevents DMBA-Induced Mammary Tumor Formation. Lab Invest 2004; 84:989-998

24. Russo J, Russo I. Atlas and histologic classification of tumors of the rat mammary gland. J Mammary Gland Biol Neoplasia 2000; 5:187-200

25. Swanson S, Guzman R, Christov K, Miyamoto S, Nandi S. Pituitary-isografted mice are highly susceptible to MNU-induced mammary carcinogenesis irrespective of the level of alveolar differentiation. Carcinogenesis 1994; 15:1341-1346

26. Thordarson G, Van Horn K, Guzman R, Nandi S, Talamantes F. Parous rats regain high susceptibility to chemically induced mammary cancer after treatment with various mammotropic hormones. Carcinogenesis. 2001; 22:1027-1033

27. Markey C, Coombs M, Sonnenschein C, Soto A. Mammalian development in a changing environment: exposure to endocrine disruptors reveals the developmental plasticity of steroid-hormone target organs. Evol Dev 2003; 5:67-75

28. Fielden M, Fong C, Haslam S, Zacharewski T. Normal mammary gland morphology in pubertal female mice following in utero and lactational exposure to genistein at levels comparable to human dietary exposure. Toxicol Lett 2002; 133:181-191

29. Lewis B, Hudgins S, Lewis A, Schorr K, Sommer R, Peterson R, 
Int. J. Biol. Sci. 2007, 3

Flaws J, Furth P. In utero and lactational treatment with 2,3,7,8-tetrachlorodibenzo-p-dioxin impairs mammary gland differentiation but does not block the response to exogenous estrogen in the postpubertal female rat. Toxicol Sci 2001; 62:46-53 